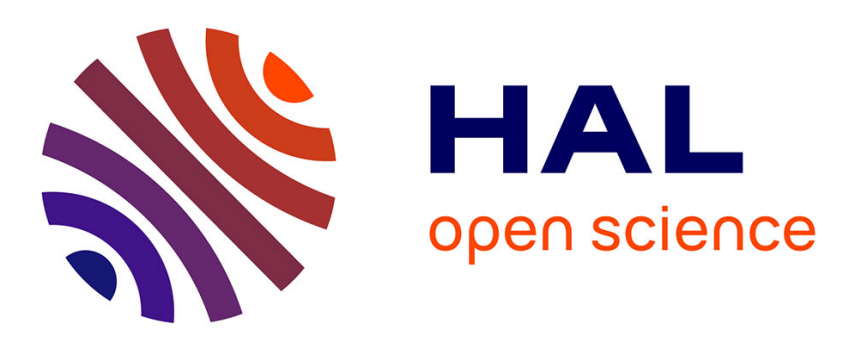

\title{
Enhancement of Compton camera images reconstructed by inversion of a conical Radon transform
}

\author{
Voichita Maxim
}

\section{To cite this version:}

Voichita Maxim. Enhancement of Compton camera images reconstructed by inversion of a conical Radon transform. Inverse Problems, 2019, 35 (1), 10.1088/1361-6420/aaecdb . hal-01932981

\section{HAL Id: hal-01932981 \\ https://hal.science/hal-01932981}

Submitted on 23 Nov 2018

HAL is a multi-disciplinary open access archive for the deposit and dissemination of scientific research documents, whether they are published or not. The documents may come from teaching and research institutions in France or abroad, or from public or private research centers.
L'archive ouverte pluridisciplinaire HAL, est destinée au dépôt et à la diffusion de documents scientifiques de niveau recherche, publiés ou non, émanant des établissements d'enseignement et de recherche français ou étrangers, des laboratoires publics ou privés. 


\title{
Enhancement of Compton camera images reconstructed by inversion of a conical Radon transform
}

\author{
Voichiţa Maxim \\ Université de Lyon, CNRS, Inserm \\ INSA-Lyon, CREATIS, UMR5220, U630, F-69621, Villeurbanne, France \\ E-mail: voichita.maxim@creatis.insa-lyon.fr
}

\begin{abstract}
We present a new inversion formula for a weighted conical Radon transform modelling Compton camera data. The formula exploits a large proportion of the acquired events and is easy to implement into fast algorithms. We give for it two equivalent formulations relying on known properties of the two-dimensional Radon transform and we test a semi-iterative algorithm for one of them. From a practical point of view, methods robust to measurement noise and to low number of events are required. We show that adding a constraint on the total variation of the final image strongly improves the results. We illustrate our arguments with Monte-Carlo simulated data in both low and realistic noise configurations.
\end{abstract}

Keywords: image reconstruction, conical Radon transform, Compton transform, analytic algorithms, Compton camera, total variation

Submitted to: Inverse Problems

\section{Introduction}

The recent interest in the conical Radon transforms was driven by the development of Compton cameras for medical and homeland security imaging applications. The idea of using Compton scattering of $\gamma$-rays as a mean to detect their incoming direction and to exploit this information for imaging was first proposed in the early 1970s independently by two groups, Schönfelder et al [1] and Todd et al [2] in the fields of $\gamma$-ray astronomy and nuclear medicine, respectively. This new technology was successfully implemented in the COMPton TELescope COMPTEL, born in 1991 on a satellite Compton GammaRay Observatory for nine years. Other projects of cameras boarded on stratospheric balloon flights were realized since then. The first tests for nuclear medicine application were reported in 1983 by Singh and Doria [3]. With the technological progress, medical applications could in a near future benefit from the large acceptance angle and the large energy spectrum allowed by the Compton imaging devices. Nowadays, the application 
fields of Compton cameras were extended to imaging in homeland security and nuclear decommissioning $([4,5,6,7])$.

The guiding thread of this study is to keep as close as possible to real applications, where the incompleteness of the data set and the acquisition noise reduce the quality of the images. Section 2 is devoted to the introduction of the main concepts employed in the paper. It contains a brief presentation of the Compton camera followed by a short stateof-the-art on conical Radon transforms, an introduction to total variation denoising ([8]) and to the Simultaneous Iterative Reconstruction Technique (SIRT, [9]). In section 3 we derive an inversion formula for a class of weighted conical Radon transforms, for cones with vertices in a plane (the scatterer) and arbitrary axis directions and half-opening angles. The filtered backprojection inversion formula we propose is closely related to the one derived in [10]. We propose three methods for the numerical computation of the inverse, two of them being analytic and the third semi-iterative. As the noise and the low number of acquired events lead to poor reconstructed images, the three of them are associated with total variation denoising or regularization. Finally we evaluate the methods with Monte-Carlo simulated data reproducing the acquisition of a complex shaped source with a finite-size detector. Two levels of uncertainties on the position and energy measurements are considered in the simulation. Section 4 describes the data, generated with a Monte-Carlo software from nuclear physics. In section 5 we show some numerical examples. We demonstrate that the methods can reconstruct the images from statistically distributed counts, but with a resolution depending on the geometry of acquisition and on the noise level.

\section{Introduction to Compton camera imaging and to total variation denoising}

In this section we introduce the principle of Compton camera imaging and we give a concise state-of-the-art on conical Radon transforms and their inverses. We also recall some widely used techniques that will be used in the next sections. They are respectively the Simultaneous Iterative Reconstruction Technique (SIRT, [9]) that is largely employed for tomographic reconstruction and the total variation (TV) denoising methodology originally introduced by Rudin et al in [8].

\subsection{Compton scattering and conical projections}

In nuclear medicine imaging Compton cameras might eventually surpass the collimated Anger cameras and allow either reduction of the radio-tracer dose injected to the patient or reduction of the acquisition time ([11]). In other medical applications where $\gamma$ imaging serves to the monitoring of the treatment, the number of emitted photons is too small to allow three dimensional imaging with collimated cameras. Additionally, the collimators hardly cope with a large energy spectrum. For this reasons, applications as treatment monitoring in proton therapy or selective internal radiation therapy are even of more 
interest but also more challenging (see e.g., $[12,13,14,15,16]$ ).

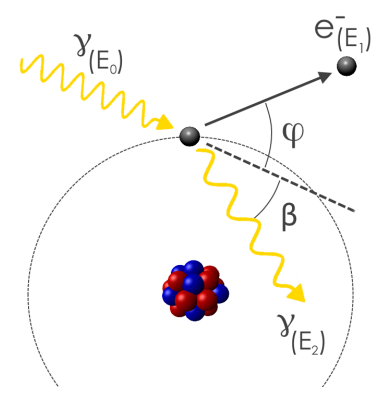

Figure 1: Inelastic scattering of a photon on an electron. A part of the energy of the photon is transferred to the electron and the last one is ejected from the atom's shell. In the simplistic model of the electron at rest the two angles, $\beta$ and $\varphi$, are uniquelly determined by the energies.

Compton cameras detect $\gamma$ rays that interact with the detector at least twice. The first interaction should be a Compton scattering, where a part of the energy of the ray is transferred to the recoiling electron (see figure 1). The last one deposits its energy in the detector, making possible to identify its position $V_{1}$ and to measure the energy $E_{1}$ lost by the photon. The $\gamma$ particle should further interact at least once at some point $V_{2}$. When the energies $E_{1}$ of the recoiled electron and $E_{0}$ of the $\gamma$ ray are known, the scattering angle may be deduced through the formula

$$
\cos \beta=1-\frac{m_{\mathrm{e}} c^{2} E_{1}}{\left(E_{0}-E_{1}\right) E_{0}},
$$

with $m_{\mathrm{e}}$ the mass of the electron at rest and $c$ the speed of the light. In the absence of noise, the point where the gamma ray was emitted lies on the surface of the cone having vertex at $V_{1}$, axis directed by the unitary vector $\Omega=\overrightarrow{V_{2} V_{1}} /\left\|\overrightarrow{V_{2} V_{1}}\right\|$ and half-opening angle $\beta$. Here \|\| denotes the usual Euclidean norm of a vector in the three-dimensional space. As a consequence, data acquired with a Compton camera having ideal position and energy resolutions use to be modelled as integrals of the weighted intensity of the source on conical surfaces. These models are gathered under the name of weighted conical Radon transforms, cone transforms or Compton transforms.

\subsection{The weighted conical Radon transform}

More precisely, the weighted conical Radon transform use to be defined as follows. For some given cone, let us denote $\alpha \in[0, \pi]$ and $\delta \in(-\pi, \pi]$ the polar and azimuthal angles of the axis direction $\boldsymbol{\Omega}, \mathbf{u} \in \mathbb{R}^{3}$ the vector of coordinates of its vertex and $\beta \in(0, \pi)$ its half-opening angle. The cone may then be defined as the set

$$
C(\mathbf{u}, \alpha, \delta, \beta)=\left\{\mathbf{v} \in \mathbb{R}^{3}:(\mathbf{v}-\mathbf{u}) . \Omega=\|\mathbf{v}-\mathbf{u}\| \cos \beta\right\},
$$

and the projection associated to it as

$$
\mathscr{C}_{\alpha, \delta, \beta}^{d} f(\mathbf{u})=\int_{C(\mathbf{u}, \alpha, \delta, \beta)} f(\mathbf{v}) \frac{1}{\|\mathbf{v}-\mathbf{u}\|^{d}} d \mathbf{v}
$$




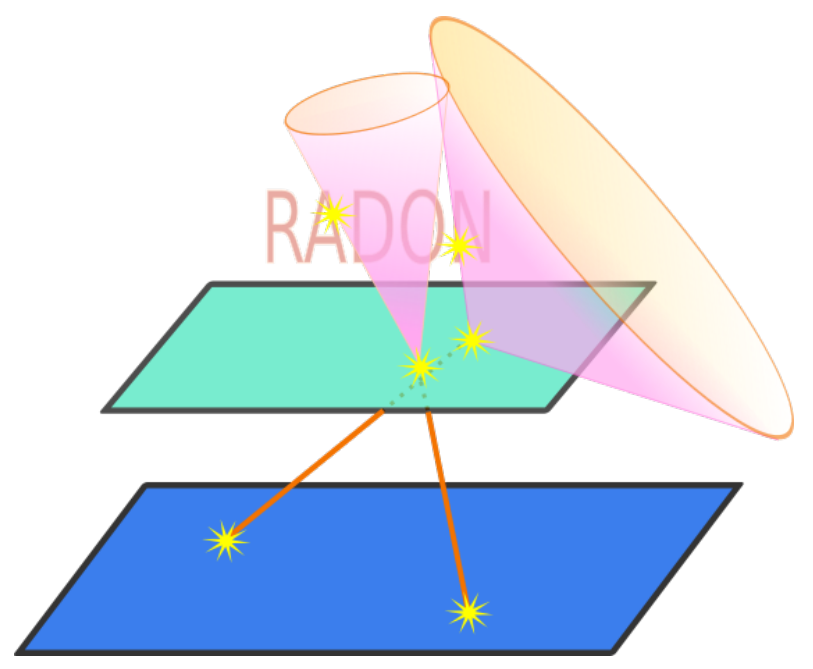

Figure 2: Schematic representation of a Compton camera composed of a scatterer (green) and an absorber (blue). The source is represented as the word RADON. Photons emitted from the source are first scattered then absorbed in the detector, and the position where they were emitted can only be determined at this stage as belonging to a conical surface.

where $f$ is the intensity function and $d$ use to be an integer. The weighted conical Radon transform of a function $f$ integrable with respect to the measure $\|\mathbf{v}-\mathbf{u}\|^{-d} d \mathbf{v}$ over almost all cones with parameters in some manifold $\mathscr{M} \in \mathbb{R}^{3} \times[0, \pi] \times(-\pi, \pi] \times(0, \pi)$, is the application

$$
(\mathbf{u}, \alpha, \delta, \beta) \in \mathscr{M} \mapsto \mathscr{C}_{\alpha, \delta, \beta}^{d} f(\mathbf{u}) .
$$

A first restriction to some $\mathscr{M}$ may be imposed by the integrability condition. A second restriction comes from the particularity of the conical Radon transform to be redundant, since the dimension of the data space is larger than the dimension of the image space. Consequently, its inversion requires to select a sub-set from the data and, when possible, to average several inverses in order to reduce the noise.

In the last years some inversion methods accompanied by adapted selection techniques were developed. In [17], Basko et al have shown that the three-dimensional Radon projections on planes containing a given point can be calculated from the set of projections on cones having the vertex at that point, arbitrary axis direction and given constant half-opening angle. Cone-beam projections (also called divergent-beam projections) can be evaluated from conical projections having the same vertex (see e.g., the works of L. Parra [18] and Tomitani and Hirasawa [19]). Detectors placed on a line were considered by Jung and Moon in [20].

The projections on cones with axes perpendicular to some surface provide a complete set. Inversion formulas in such configurations were given by Cree and Bones [21] and M. Haltmeier [22] for the plane, Schiefeneder and Haltmeier [23] for the sphere, S. Moon [24] for surfaces of revolution and Moon and Haltmeier [25] for the cylinder. Inversion methods that restrict the useful set of projections to cones with axes perpendicular on a manifold will generally neglect a large part of the acquired data and 
thus provide very noisy images. A particular case where they may be of interest is the one of small detectors that move around the patient (see e.g., [26]). Such detectors can anyway measure projections only for small values of $\alpha$.

When the values of the projections are known for single axis direction and halfopening angle but for all vertices $u \in \mathbb{R}^{3}$, the mapping (4) can be seen as a convolution of $f$ with a distribution supported on the cone. Deconvolution formulas were given by Gouia-Zarrad and Ambartsoumian ([27]), F. Terzioglu ([28]) and V. Palamodov ([29]). The inconvenient of this approach for the application to Compton camera is that measurements are required in all points of the space, including inside the source. This is obviously impossible to realize since the detectors are always placed outside the body. The methods proposed by B. Smith ([30]), Kuchment and Terzioglu ([31, 32]), Terzioglu et al ([33]) and one of the methods given by V. Palamodov in [29] allow to select the points of some manifold $\mathscr{M} \subset \mathbb{R}^{3}$ that do not intersect the source. The manifolds $\mathscr{M}$ are characterised by some admissibility (see [31, 32]) or completness (see [30]) conditions that are very similar to each other, with specificities related to the inversion formula.

Connected to the topic we discuss here is the inversion of the cumulative (or compound) conical Radon transform addressed e.g., in [34] by Nguyen and Truong. This transform arises in nuclear medicine imaging when one wants to make an image with the photons scattered inside the patient. The differences in the inversion methods are however important and the problem is more complicated in the sense that the projections are not measured individually.

\subsection{Conical Radon transform for cones with vertices in a plane}

A slightly different class of weighted conical transforms was analysed in [35] under the name of Compton transforms. Their specificity is to employ a weight different from (3), weight that accounts for the incident angle of the incoming particle, and to consider the set of cones with vertices in a plane. Two transforms from the class differ either by the polar angle $\alpha$ or by the half-opening angle $\beta$ and can be averaged together for noise variance reduction as shown in [10]. The main result of [35] is the central-slice theorem from section 4.1, proving the invertibility of the transform. Derived afterwards in [10], the associated filtered back-projection formula benefits from a relative simplicity and also from the fact that conical projections with axis directions not orthogonal to the detector are allowed.

To ensure uniqueness of the inverse, it is assumed that all the projections on cones with vertices in some given plane are measured. This completeness condition is obviously not verified in practice. The method can still give reasonably good results for Monte-Carlo simulated data, providing addition of some smoothing. In [10], this was obtained by frequency damping. In this paper we propose to use total variation either for denoising the solution or as a regularization step associated with an iterative algorithm. 


\subsection{Total variation regularization}

Originally proposed by Rudin et al in [8], total variation penalty function is recommended for denoising piecewise constant images, as it promotes smoothness while still conserving sharp edges. Total variation may also improve tomographic reconstruction of images from low-dose acquisitions (see e.g., [36]), or from limitedangle data (see e.g., [37, 38]).

We suppose without loose of generality that the volume is a cube divided in $N^{3}$ voxels, rearranged in a vector $\mathbf{g}$, and we note $X=\mathbb{R}^{3 N}$. The volume is transformed through a linear map of matrix $R$ and the measurements $\mathbf{p}$ are noisy realizations of $R \mathbf{g}$. We search for $\mathbf{g}$ solution of the regularized problem:

$$
\underset{\mathbf{g} \in X}{\arg \min }\left\{\frac{1}{2}\|R \mathbf{g}-\mathbf{p}\|_{2}^{2}+\mu T V(\mathbf{g})\right\},
$$

with $\mu \geq 0$ the regularization parameter and $T V$ the total variation norm. For $\Omega$ an open subset of $\mathbb{R}^{3}$, the total variation of a function $g \in L_{1}(\Omega)$ is defined by:

$T V(g)=\sup \left\{\int_{\Omega} g(x) \operatorname{div}(\varphi(x)) d x: \varphi \in C_{c}^{1}\left(\Omega ; \mathbb{R}^{3}\right),\|\varphi(x)\| \leq 1 \forall x \in \Omega\right\}$.

If $g$ has gradient $\nabla g \in L_{1}(\Omega)$, then $T V(g)=\int_{\Omega}\|\nabla g(x)\| d x$. The discrete form of the total variation uses to be written as:

$$
T V(\mathbf{g})=\sum_{1 \leq i, j, k \leq N}\left\|(\nabla \mathbf{g})_{i, j, k}\right\|
$$

and is not differentiable in zero. Let us put $Y=\left(\mathbb{R}^{3}\right)^{3 N}$. An equivalent expression for the discrete total variation is:

$T V(\mathbf{g})=\sup \left\{\langle\boldsymbol{\varphi}, \nabla \mathbf{g}\rangle_{Y}: \varphi \in Y\right.$ such that $\left\|\varphi_{i, j, k}\right\| \leq 1$ for all $\left.1 \leq i, j, k \leq N\right\}$,

where $\langle\boldsymbol{\varphi}, \boldsymbol{\psi}\rangle_{Y}=\sum_{i, j, k}\left(\sum_{\ell=1}^{3} \varphi_{i, j, k}^{\ell} \psi_{i, j, k}^{\ell}\right)$ is the Euclidean dot product in $Y$. With the appropriate definition of the discrete divergence, $\langle\boldsymbol{\varphi}, \nabla \mathbf{g}\rangle_{Y}=-\langle\operatorname{div} \boldsymbol{\varphi}, \mathbf{g}\rangle_{X}$. In this work we rely on the notations from [39], and we apply the Chambolle's algorithm proposed therein to solve the denoising problem when $R$ is the identity matrix.

When $R$ is not the identity matrix a splitting approach can be used, with a gradient descent step that decreases the value of the data fidelity term $\|R \mathbf{g}-\mathbf{p}\|_{2}$ and a TVdenoising step that smooths the solution. This approach was previously applied by Beck and Teboulle in [40] for deconvolution problems and the gradient descent algorithm was used for the first task. When $R$ stands for the discrete Radon transform, the SIRT algorithm may be applied. SIRT may be considered as a weighted gradient descent algorithm with more stable results.

\subsection{The SIRT algorithm for the inversion of the Radon transform}

In this paper we make use of the iterative inversion of the Radon transform. The Simultaneous Iterative Reconstruction Technique (SIRT, [9]) is one of the algorithms widely employed for this task. Let $R$ be the system matrix describing the discrete Radon 
transform in $2 \mathrm{D}$ or the X-ray transform in $3 \mathrm{D}, \mathbf{p}$ the vector of data and $\mathbf{g}$ the vector containing all the voxels of the volume we wish to reconstruct. The SIRT algorithm starts from an initial guess $\mathbf{g}^{(0)}$ that may be taken as the null vector or the filtered back-projection solution then iterates following the formula:

$$
\mathbf{g}^{(k+1)}=\mathbf{g}^{(k)}-\lambda\left(R^{*}\left[\frac{R \mathbf{g}^{(k)}-\mathbf{p}}{R \mathbf{1}}\right]\right) /\left(R^{*} \mathbf{1}\right),
$$

where $\lambda$ is a descent step, $\mathbf{1}$ is a vector of ones and $R^{*}$ is the Hermitian conjugate of $R$. The divisions of vectors is done component-wise. In all our numerical tests we take $\lambda=0.2$. The SIRT algorithm may give reconstructions more precise than the filtered back-projection, but when the data is very noisy a regularization of the solution is necessary. The best way to introduce this regularization is to add it as a penalty term in the minimization problem, as explained in section 2.4.

\section{Theoretical and numerical inversion of the conical Radon transform}

In this section we give an analytic inversion formula for the transform defined in equation (3) for $d=1$, where the integral of the intensity of the source is weighted by the inverse of the distance from the source point to the vertex of the cone. We propose three equivalent formulations for the inverse and discuss them from a numerical and discrete point of view. One of the methods we propose is semi-iterative, in the sense that a part of the computation, aiming to transform the Compton data in two-dimensional Radon data, is done analytically. Then an iterative algorithm alternating SIRT ([9]) iterations and TV denoising is applied in order to calculate a regularized inverse of the Radon transform. The other methods are analytical and based on the commutativity of the Radon transform with differentiation.

\subsection{Discussion on the Compton camera data modelling}

We consider in this work cameras with planar detectors (see figure 2). In the scope of a simpler presentation and without losing generality, hereafter we consider a camera containing a single scatterer layer placed at altitude $z_{s}=0$ and an absorber placed at some altitude $z_{a}<0$. When the scatterer has several layers, a conical transform should be defined for each layer. The intensity $f$ is then calculated as the mean of their inverses. We suppose that the probabilities for an incident $\gamma$ ray to be Compton scattered in the first detector and then completely absorbed in the second detector are both equal to one, so that these probabilities do not appear in the model. The differential cross section of a $\gamma$ photon having initial energy $E_{0}$ and scattered by an angle $\beta$, is given by the KleinNishina formula $([41])$, and is denoted by $K\left(\beta, E_{0}\right)$. For a given cone, let $\mathbf{u} \in \mathbb{R}^{2} \times\{0\}$ be the position vector of the vertex and $\Omega=(\sin \alpha \cos \delta, \sin \alpha \sin \delta, \cos \alpha)$ the direction vector of its axis, where $\alpha$ and $\delta$ are its polar and azimuthal angles (see figure 3 (a)).

When a source of $\gamma$ particles having intensity distribution represented by a function

$f: \mathbb{R}^{3} \rightarrow \mathbb{R}$ is observed by a Compton camera, the fraction of events recorded with 
parameters $(\mathbf{u}, \alpha, \delta, \beta)$ was considered in [35] proportional to

$$
\begin{aligned}
\mathscr{C}_{\alpha, \delta, \beta} f(\mathbf{u}) & =K\left(\beta, E_{0}\right) \cos \alpha \int_{C(\mathbf{u}, \alpha, \delta, \beta)} f(\mathbf{v}) \cos \theta d \mathbf{v} \\
& =K\left(\beta, E_{0}\right) \cos \alpha \int_{C(\mathbf{u}, \alpha, \delta, \beta)} f(\mathbf{v}) \frac{z}{\|\mathbf{v}-\mathbf{u}\|} d \mathbf{v},
\end{aligned}
$$

where $\mathbf{v}=(x, y, z)$ is a vector from $\mathbb{R}^{3}$ and $\theta$ is the polar angle of the vector $\mathbf{v}-\mathbf{u}$. The factors $\cos \theta$ and $\cos \alpha$ from (10) account for the arbitrary incidence angle of the $\gamma$ ray on the scatterer and absorber, respectively. For any given parameters $\alpha, \delta, \beta$, with $\alpha \in[0, \pi], \beta \in(0, \pi)$ and $\delta \in(-\pi, \pi]$, the function $\mathscr{C}_{\alpha, \delta, \beta} f: \mathbb{R}^{2} \times\{0\} \rightarrow \mathbb{R}$ was called in [35] a Compton projection of $f$ (see figure $3(\mathrm{~b})$ ). In order to keep the apelation from [35] and to distinguish it from other conical Radon transforms from the literature, the integral transform $\mathscr{C}$ operating on functions $f: \mathbb{R}^{3} \rightarrow \mathbb{R}$ is hereafter referred to as the Compton transform.

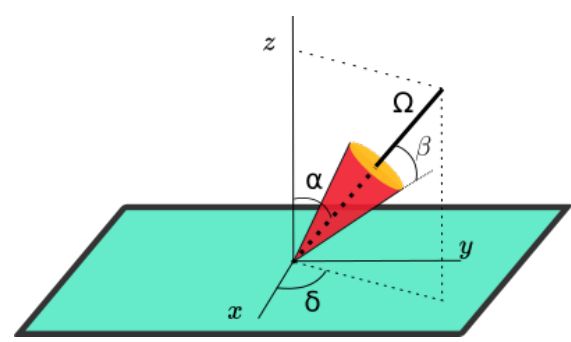

(a)

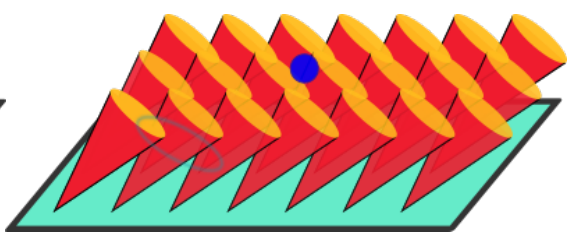

(b)

Figure 3: (a) Parameterization of the Compton cone. (b) Construction of the Compton projection $\mathscr{C}_{\alpha, \delta, \beta}$. The Compton projection is the image drawn on the surface of the scatterer by the vertices of the cones with parameters $\alpha, \delta, \beta$ that intersect the source. The intensity of the print is the integral from (10).

In figure 4 are represented a deterministic Compton projection and its counterpart from a Monte-Carlo simulation. The source is a small sphere. The deterministic projection from (a) was calculated in each pixel of the scatterer following equation (10). In (b) a Compton camera acquisition with a small spherical source emitting $\gamma$ photons was simulated with the software MEGAlib (The Medium-Energy Gamma-ray Astronomy library, [42]), software based on the nuclear physics simulation library Geant4 [43]. The simulation in figure 4 accounts for Doppler broadening i.e., small errors on the measured energies produced when the photon is scattered on an electron which is not at rest. In this example, the positions of the hits and the sum of energies are exact.

In (10) the weight $\cos \theta$ only accounts for the incidence angle of the $\gamma$ ray on the scatterer. In a series of papers (see e.g., [44]), Wilderman et al suggested that the weight should also depend on the distance $\|\mathbf{v}-\mathbf{u}\|$ through the solid angle subtended by the element of detector at the source. The model,

$$
\mathscr{C}^{*}{ }_{\alpha, \delta, \beta} f(\mathbf{u})=K\left(\beta, E_{0}\right) \int_{C(\mathbf{u}, \alpha, \delta, \beta)} f(\mathbf{v}) \frac{\cos \theta}{\|\mathbf{v}-\mathbf{u}\|^{2}} d \mathbf{v},
$$




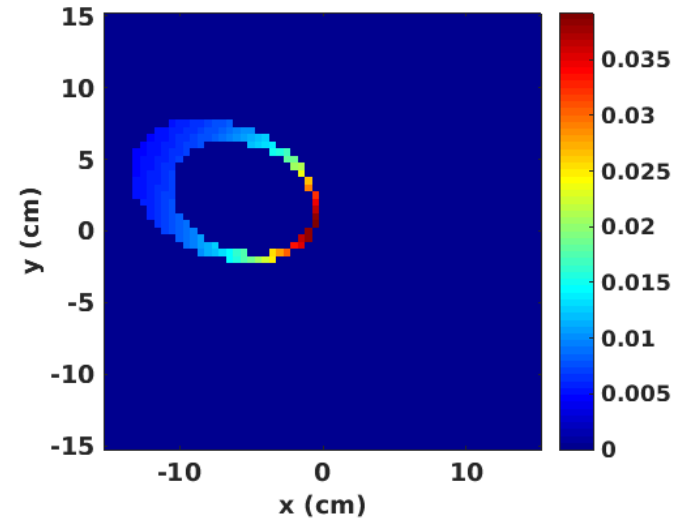

(a)

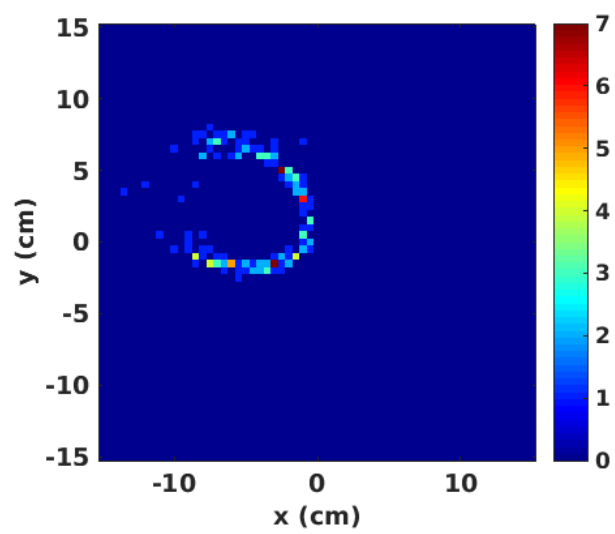

(b)

Figure 4: Example of Compton projections of a small spherical source. In (a) the projection is calculated deterministically following (10). In (b) a Monte Carlo simulation of the same detector-source configuration is shown. The model serving for the deterministic projection (a) only approximatelly describes the data in (b).

was recently validated against Monte-Carlo simulations ([45]). As the source is supposed to lie in the half-space $z>0$, the function $f$ is zero for values of $\mathbf{v}$ close to $\mathbf{u}$. Thus there is no singularity in the expression under the integral (11). As we shall show in section 3.2 , the inversion formula given in [10] for (10) can be adapted easily to $d=1$ and the transform

$$
\mathscr{C}_{\alpha, \delta, \beta}^{1} f(\mathbf{u})=K\left(\beta, E_{0}\right) \int_{C(\mathbf{u}, \alpha, \delta, \beta)} f(\mathbf{v}) \frac{1}{\|\mathbf{v}-\mathbf{u}\|} d \mathbf{v}
$$

which is closer to the model suggested by the Monte Carlo simulations and in practice seems to give better results.

\subsection{Inversion of the conical Radon transform}

In this section we derive a formula allowing to calculate the source intensity from the conical Radon projections (12). To avoid technical difficulties throughout the following, we assume $f$ to be Schwartz function supported in the upper half space.

Let $\left(\mathbf{e}_{1}, \mathbf{e}_{2}, \mathbf{e}_{3}\right)$ be the standard basis of $\mathbb{R}^{3}$. The vectors $\mathbf{d}_{1}=(\cos \delta, \sin \delta, 0)$, $\mathbf{d}_{2}=(-\sin \delta, \cos \delta, 0)$ and $\mathbf{e}_{3}$ also form an orthonormal basis of $\mathbb{R}^{3}$, defining a reference frame Otsz obtained by rotation of $O x y z$ about the $O z$ axis. Let us consider the cone $C\left(\mathbf{u}_{0}, \alpha, \delta, \beta\right)$ with $\mathbf{u}_{0}=t_{0} \mathbf{d}_{1}+s_{0} \mathbf{d}_{2}$. Since the source belongs to the half-space $z>0$, for a single-layer scatterer one should always have $\alpha \in\left[0, \frac{\pi}{2}\right)$. No restriction apply on $\delta$, which is taken in the interval $(-\pi, \pi]$. Physically, the Compton angle $\beta$ can take any value in $(0, \pi)$. However the parametrization we choose hereafter for the cone constrains to keep only the Compton projections with $\alpha+\beta<\frac{\pi}{2}$, leading to a selection of the cones that intersect the scatterer in a single point, the vertex. For such admissible values of 
$\alpha$ and $\beta$ one has $\cos ^{2} \alpha-\sin ^{2} \beta>0$. We define:

$$
\begin{aligned}
& a(\alpha, \beta)=\frac{\sin \beta \cos \beta}{\cos ^{2} \alpha-\sin ^{2} \beta}, \\
& b(\alpha, \beta)=\frac{\sin \beta}{\sqrt{\cos ^{2} \alpha-\sin ^{2} \beta}}, \\
& c(\alpha, \beta)=\frac{\sin \alpha \cos \alpha}{\cos ^{2} \alpha-\sin ^{2} \beta} .
\end{aligned}
$$

The intersection of the cone with a horizontal plane at some given altitude $z>0$ is an ellipse that may be described by the parametric equation:

$$
\left\{\begin{array}{ll}
t=t_{0}+z c(\alpha, \beta) & +z a(\alpha, \beta) \cos \varphi \\
s=s_{0} & +z b(\alpha, \beta) \sin \varphi
\end{array}, \quad \varphi \in(-\pi, \pi] .\right.
$$

With the cone thought of as a stack of ellipses, its parametric equation may then be readily obtained. The surface integral (12) at a point from the scatterer having coordinates $\mathbf{u}_{0}=t_{0} \mathbf{d}_{1}+s_{0} \mathbf{d}_{2}$, may then be expressed as

$$
\begin{aligned}
& \mathscr{C}_{\alpha, \delta, \beta}^{1} f\left(t_{0} \mathbf{d}_{1}+s_{0} \mathbf{d}_{2}\right)=K\left(\beta, E_{0}\right) b(\alpha, \beta) \\
& \times \int_{0}^{\infty} \int_{0}^{2 \pi} f\left(\left(t_{0}+z c(\alpha, \beta)+z a(\alpha, \beta) \cos \varphi\right) \mathbf{d}_{1}+\left(s_{0}+z b(\alpha, \beta) \sin \varphi\right) \mathbf{d}_{2}+z \mathbf{e}_{3}\right) d \varphi d z .
\end{aligned}
$$

Hereafter we make use of the two-dimensional Radon transform, that associates to a function $g: \mathbb{R}^{2} \rightarrow \mathbb{R}$ with bounded support a set indexed on the parameter $\delta \in(-\pi, \pi]$ of line integrals $\mathscr{R}_{\delta}$, defined as:

$$
\forall s \in \mathbb{R}, \quad \mathscr{R}_{\delta} g(s)=\int_{-\infty}^{\infty} g(s \cos \delta+t \sin \delta, s \sin \delta-t \cos \delta) d t .
$$

We note $f_{z}:(x, y) \in \mathbb{R}^{2} \mapsto f(x, y, z) \in \mathbb{R}$ the restriction of $f$ to some horizontal plane. For $\tau \geq 0$ and $\delta \in(-\pi, \pi]$, we define the integral transform given for all $s \in \mathbb{R}$ by:

$$
\mathscr{P}_{\tau, \delta} f(s)=\tau \int_{0}^{\infty} \int_{-\pi}^{\pi} \mathscr{R}_{\delta+\frac{\pi}{2}} f_{z}(s+z \tau \sin \varphi) d \varphi d z .
$$

Simple calculations starting from (17) and (18) allow to show that the transforms $\mathscr{P}$ and $\mathscr{C}^{1}$ are related to each other by

$$
\mathscr{P}_{\tau, \delta}=\frac{1}{K\left(\beta, E_{0}\right)} \mathscr{R}_{\delta+\frac{\pi}{2}} \mathscr{C}_{\alpha, \delta, \beta}^{1}
$$

whenever $\tau=b(\alpha, \beta)$. The transform $\mathscr{P}$ produces a data set included in a three dimensional space, indexed by $\tau>0, \delta \in(-\pi, \pi]$ and $s \in \mathbb{R}$. The required data set may be further reduced by taking into account the identity

$$
\mathscr{R}_{\delta+\frac{\pi}{2}} \mathscr{C}_{\alpha, \delta+\pi, \beta}^{1}=\mathscr{R}_{\delta+\frac{\pi}{2}} \mathscr{C}_{\alpha, \delta, \beta}^{1},
$$

meaning that from the point of view of the transform $\mathscr{P}$, the projections $\mathscr{C}_{\alpha, \delta+\pi, \beta}^{1}$ and $\mathscr{C}_{\alpha, \delta, \beta}^{1}$ share the same information.

Taking the Fourier transform in (19) leads to:

$$
\widehat{\mathscr{P}_{\tau, \delta} f}(\sigma)=2 \pi \tau \int_{0}^{\infty} \widehat{\mathscr{R}} \delta+\frac{\pi}{2} f_{z}(\sigma) J_{0}(2 \pi z \tau \sigma) d z,
$$


where $J_{0}$ is the zero-order Bessel function of the first kind. Then, by the projection-slice theorem applied to the Radon projection and for $d_{2}=(-\sin \delta, \cos \delta, 0)$ we get:

$$
\widehat{\mathscr{P}_{\tau, \delta} f}(\sigma)=2 \pi \tau \int_{0}^{\infty} \frac{1}{z} \widehat{f}_{z}\left(\sigma d_{2}\right) J_{0}(2 \pi z \tau \sigma) z d z .
$$

In (23), $f_{z}=0$ for $z$ close to the origin. Formulas (22), (23) are valid for all Schwartz functions $f$ supported in the upper half space and both appearing integrals are absolutely convergent. We recognize in (23) the Hankel transform of $\widehat{f}_{z} / z$. Finally the inversion of the Hankel transform leads to

$$
\widehat{f_{z}}\left(\sigma d_{2}\right)=2 \pi z \sigma^{2} \int_{0}^{\infty} \widehat{\mathscr{P}_{\tau, \delta} f}(\sigma) J_{0}(2 \pi z \tau \sigma) d \tau,
$$

and to the reconstruction formula:

$f(x, y, z)=2 \pi z \int_{0}^{\pi} \int_{0}^{\infty}\left(\int_{-\infty}^{\infty} \widehat{\mathscr{P}_{\tau, \delta} f}(\sigma) J_{0}(2 \pi z \tau \sigma)|\sigma|^{3} e^{2 i \pi \sigma(-x \sin \delta+y \cos \delta)} d \sigma\right) d \tau d \delta$.

Note that when $b(\alpha, \beta)=\tau>0$, either $\alpha$ or $\beta$ may be calculated from (14) as function of the other parameter through the relation:

$$
\tau \cos \alpha=\sqrt{1+\tau^{2}} \sin \beta,
$$

since both applications $\beta \in\left(0, \frac{\pi}{2}-\alpha\right) \mapsto b(\alpha, \beta) \in \mathbb{R}_{+}^{*}$, for some given $\alpha \in\left[0, \frac{\pi}{2}\right)$, and $\alpha \in\left[0, \frac{\pi}{2}-\beta\right) \mapsto b(\alpha, \beta) \in \mathbb{R}_{+}^{*}$, for some given $\beta \in\left(0, \frac{\pi}{2}-\alpha\right)$, are one-to-one. This means that in $(25)$, the projections $\mathscr{P}_{\tau, \delta}$ can be calculated as the mean of an infinity of conical projections, one for each polar angle $\alpha$ or one for each Compton angle $\beta$.

Numerically, the reconstruction can be done slice by slice (following the direction orthogonal to the camera) as follows. The Radon projections of the Compton projections are filtered in the Fourier domain with a filter depending on the parameter $\tau$, related to the cone pattern generating the projection, and on the altitude $z$. The filtered projections are then integrated on $\tau$ then back-projected on lines in each horizontal slice. The filtering in the Fourier domain relies on the evaluation of the Bessel function $J_{0}$ on a grid sufficiently fine to avoid aliasing as much as possible. Consequently, zeropadding of the projections is necessary implying intensive use of the memory when all projections are processed simultaneously.

The evaluation of the reconstruction formula may produce very noisy results as the reconstruction filter $|\sigma|^{3}$ amplifies the noise in the data while emphasizing high frequencies. A practical yet insufficient solution is the damping of the higher frequencies during the reconstruction. Some post-filtering remains necessary and may be obtained by total variation denoising.

\subsection{Alternative formulations of the inverse Compton transform}

In a tempered distribution sense the Bessel function $\sigma \mapsto J_{0}(2 \pi z \tau \sigma)$ is the Fourier transform of

$$
k_{z, \tau}(s)=\frac{1}{2 \pi} \frac{2 \operatorname{rect}\left(\frac{s}{2 z \tau}\right)}{\sqrt{(z \tau)^{2}-s^{2}}},
$$


where rect denotes the rectangular function. With ramp the impulse response of the ramp filter $\sigma \mapsto|\sigma|$, the filtered projections may be rewritten as:

$\int_{-\infty}^{\infty} \widehat{\mathscr{P}_{\tau, \delta} f}(\sigma) J_{0}(2 \pi z \tau \sigma)|\sigma|^{3} e^{2 i \pi \sigma \mathbf{v} \cdot \mathbf{d}_{2}} d \sigma=-\frac{1}{(2 \pi)^{2}}\left(\mathscr{P}_{\tau, \delta} \star k_{z, \tau} \star \operatorname{ramp}\right)^{\prime \prime}\left(\mathbf{v} \cdot \mathbf{d}_{2}\right)$.

With the notation $g_{z, \tau, \delta}=\mathscr{P}_{\tau, \delta} \star k_{z, \tau}$ we then have

$\int_{-\infty}^{\infty} \widehat{\mathscr{P}_{\tau, \delta} f}(\sigma) J_{0}(2 \pi z \tau \sigma)|\sigma|^{2} e^{2 i \pi \sigma\left(\mathbf{v} \cdot \mathbf{d}_{2}\right)} d \delta=-\frac{1}{(2 \pi)^{2}}\left(g_{z, \tau, \delta} \star \operatorname{ramp}\right)^{\prime \prime}\left(\mathbf{v} \cdot \mathbf{d}_{2}\right)$.

The function $g_{z, \tau, \delta}$ may be calculated either in the temporal or the frequency domain. The first alternative seems preferable, because the highly oscillatory Bessel function requires a high sampling rate to limit aliasing as much as possible (see figures 5 and $6)$. This frequency domain sampling may be obtained by zero-padding the projections $\mathscr{P}_{\tau, \delta}$.

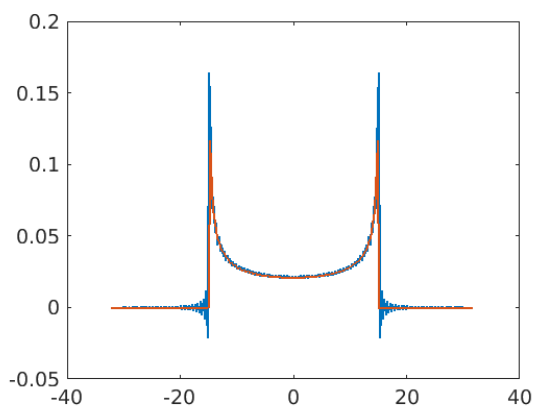

Figure 5: Inverse Fourier transform of the discretized Bessel function (blue line) compared to $k_{z, \tau}$ (red line).

Let us now put for each $s \in \mathbb{R}$,

$$
p_{z}(s, \delta)=z \int_{0}^{\infty} g_{z, \tau, \delta}(s) d \tau
$$

From (29) we then deduce that

$\int_{0}^{\infty}\left(\int_{-\infty}^{\infty} \widehat{\mathscr{P}_{\tau, \delta} f}(\sigma) J_{0}(2 \pi z \tau \sigma)|\sigma|^{3} e^{2 i \pi \sigma\left(\mathbf{v} \cdot \mathbf{d}_{2}\right)} d \delta\right) d \tau=-\frac{1}{(2 \pi)^{2} z}\left(p_{z}(\cdot, \delta) \star \operatorname{ramp}\right)^{\prime \prime}\left(\mathbf{v} \cdot \mathbf{d}_{2}\right),(31$

and finally from (25) we obtain

$$
f(x, y, z)=-\frac{1}{2 \pi}\left(\mathscr{R}^{-1}\left[\frac{\partial^{2} p_{z}}{\partial s^{2}}\right]\right)(x, y),
$$

where $\mathscr{R}^{-1}$ is the two-dimensional inverse Radon transform. We have thus shown that the image of the source may be reconstructed slice by slice as the inverse of the twodimensional Radon transform, from synthetic Radon projections calculated by postprocessing the Compton projections. From a numerical point of view, formula (32) has several advantages:

(i) It gives the possibility to use iterative methods combined with advanced filtering for the inversion of the Radon transform, still conserving a fast method compared to direct use of iterative methods on the raw Compton data; 


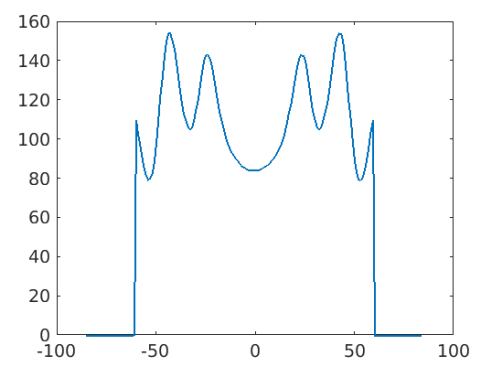

(a) raw projection $\mathscr{P}_{\tau, \delta}$

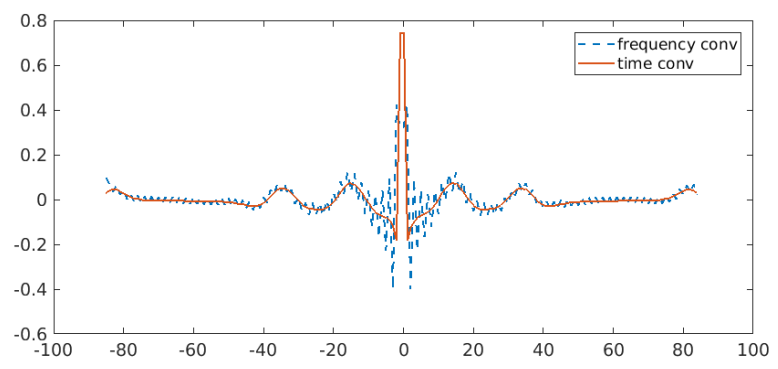

(b) second derivative of filtered projection $g_{z, \tau, \delta}$

Figure 6: (a) Example of projection $\mathscr{P}_{\tau, \delta}$, i.e., Radon projection of the Compton projection, for a source composed of three points. (b) Its filtered versions $g_{z, \tau, \delta}^{\prime \prime}$ for some arbitrary value $z$, with the convolution calculated in the frequency domain (blue doted line) and in the time domain (red continuous line). The oscillations due to aliasing that may be observed in the right-hand image are important, but this is not always the case. Aliasing is more visible for large values of $z \tau$. Since they are attenuated by the usual smoothing of projections in the reconstruction process, the influence of the filtering domain may finally have little impact on the images.

(ii) It has lower memory requirements when the convolution with the filter $k$ is done in the time domain compared to Fourier domain convolution with the highlyoscillating Bessel function;

(iii) It makes use of a more precise calculation of the filtered projections $g_{z, \tau, \delta}$ which are no more subject to aliasing. An example is shown in figure 6 .

In our experiments, only the first point appeared as important. The third point had little impact on the results, but this observation may be biased by the small distance we put between the source and the detector.

\subsubsection{Semi-iterative inversion of the Compton transform}

The images obtained from (32) may be denoised with a total variation filtering. However, post-filtering increases the projection errors and a better approach is to apply an iterative algorithm to the regularized problem as explained at the end of section 2.4. In this case we choose to solve a problem of type (5). Following the procedure described in section 2.4, SIRT steps are alternated with total variation denoising steps completed with the Chambolle's algorithm from [39]. If $\lambda$ is the descent step of SIRT and $\mu$ is the regularization parameter multiplying the TV norm in (5), the parameter of the total variation denoising should be taken equal to the product $\lambda \mu$.

\subsubsection{Analytic solution written as a second derivative}

In (32) the back-projection is preceded by the differentiation of the projections. The derivative is calculated by finite differences and this operation induces some circular blurring in the reconstructed images. 
The commutativity of the Radon transform with derivatives is a classical result (see e.g., [46]). For any $g: \mathbb{R}^{2} \mapsto \mathbb{R}$ sufficiently smooth,

$$
\mathscr{R}_{\delta}\left(\partial_{1} g\right)=(\cos \delta)\left(\mathscr{R}_{\delta} g\right)^{\prime} \quad \text { and } \quad \mathscr{R}_{\delta}\left(\partial_{2} g\right)=(\sin \delta)\left(\mathscr{R}_{\delta} g\right)^{\prime} .
$$

and when $\Delta=\partial_{11}+\partial_{22}$ is the Laplace operator,

$$
\mathscr{R}_{\delta}(\Delta g)=\left(\mathscr{R}_{\delta} g\right)^{\prime \prime} \text {. }
$$

We thus may rewrite (32) as

$$
f(x, y, z)=-\frac{1}{2 \pi} \Delta\left(\mathscr{R}^{-1} p_{z}\right)(x, y) .
$$

In the continuous case this formula is equivalent to (32) and thus to (25). In the discrete case the equivalence do not hold any more and formula (33) may produce less blurry images. Numerically, the Laplacian can be calculated by finite differences. In this work we used the del2 function from MATLAB.

\section{Description of the data}

We generated Compton camera data with the Monte-Carlo simulation software MEGAlib (The Medium-Energy Gamma-ray Astronomy library) described in [42]. This

software is composed of several modules, and is based on the Geant4 library [43]. The COSIMA module allows to simulate the interactions between the particles and the matter. The output consists in a list of interactions for each individual photon and for the secondary particles produced during these interactions. The ordering of the hits in the sequence is currently available only in simulations, as the time resolution of the detectors is too poor. In a real acquisition, the camera would provide a set of (not ordered) interactions and specific algorithms are required to reconstruct the sequence of hits.

The Monte-Carlo data may then be analysed with one of the two modules SIVAN and REVAN. The first one retrieves the ideal positions of the hits and the exact energies of the secondary particles. Only Doppler broadening may affect the measurements. Additionally the order of the interactions is known in this case and the vertex and axis of the cone are thus the true ones. When the data are processed with REVAN, errors are added both on positions and energies. These errors reverberate on the value of the scattering angle, on the position of the vertex and on the direction of the axis of the cone. The order of the interactions is determined based on kinematics considerations and may be erroneous. The first hit is eventually wrongly identified and leads to a miss-positioning of the vertex of the cone. The same may hold for the second hit, with consequences on the axis direction.

\subsection{Influence of the errors on the angular parameters}

The errors on the angular parameters of the Compton cone have important consequences on the reconstructed images regardless the algorithm. For instance in figure 7 we show 
iterative Maximum Likelihood Expectation-Maximization (MLEM) reconstructions from deterministic projections of three identical point-like sources. The algorithm was originally adapted to Compton camera imaging by Wilderman et al in [44] and here we use the implementation from [14]. In (a) the projections are simulated with $\alpha=2.5^{\circ}$ and reconstructed as they were acquired at $\alpha=0^{\circ}$. In (b), the projections for $\beta=10^{\circ}$ and $\beta=15^{\circ}$ are summed together then reconstructed as they were taken at $\beta=12.5^{\circ}$. In both cases the intensities of the sources are different after reconstruction and the blur increases as we move further from the detector. Whilst the errors on $\alpha$ produced in this experiment only an elongation of the source, the influence of the errors on $\beta$ is more critical.

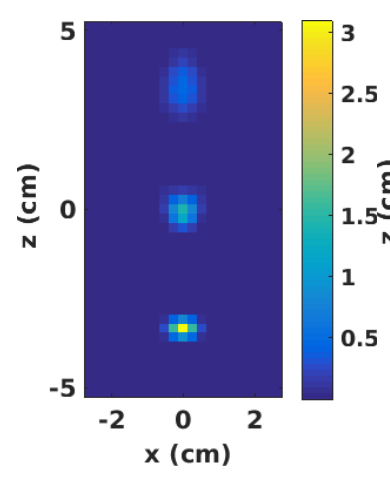

(a)

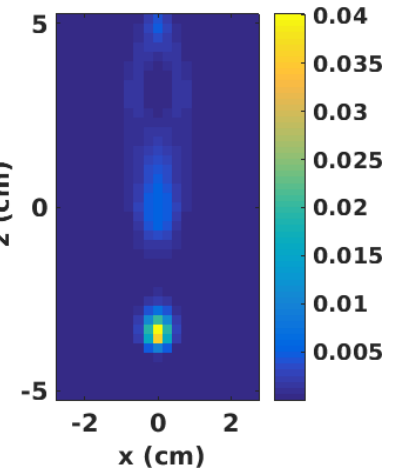

(b)

Figure 7: Image reconstructed with the Maximum Likelihood ExpectationMaximization algorithm (MLEM) from projections with (a) an error of $2.5^{\circ}$ on the axis inclination $\alpha$ and (b) an error of $2.5^{\circ}$ on the Compton scattering angle $\beta$ (see text for details). Twenty iterations of the MLEM algorithm were runned.

Figure 7 shows that the artefacts in the vertical direction are not specific to analytic reconstruction. They are not necessarily related to an erroneous modelling of the data since in this example the model from which the data is generated is perfectly known. Elongation of the source in the direction orthogonal to the camera is usual in Compton camera imaging and is often related to the small solid angle subtended by the detector at a point from the source. As it is produced by a loss of projections, it could be assimilated to a "limited angle" artefact. In this example the camera was designed to be relatively large and close to the sources. We shall thus conclude that data binning strongly reinforces the artefacts in the vertical direction.

\subsection{Compton camera simulated data}

For the purpose of evaluation of the analytic methods we simulated a Compton camera composed of six Silicon scatterer layers and one absorber composed of $\mathrm{LaBr}_{3}$ crystals. Each layer has dimensions $30 \times 30 \times 0.2 \mathrm{~cm}^{3}$ and the absorber is composed of $128^{2}$ crystals arranged in a $73 \mathrm{~cm}$-side square. Each crystal has dimensions $0.5 \times 0.5 \times 4 \mathrm{~cm}^{3}$. 
The scatterers are placed with the centres at altitudes $z \in\{-5,-6, \ldots,-10\}$ and the absorber at $z=-22$.

For the noisy data, the spatial resolution in the scatterer is about $1 \mathrm{~mm}$. In the absorber, the hit is always considered at the center of a crystal. The energy errors are randomly drawn from Gaussian distributions. In the scatterer its standard deviation is $1 \mathrm{keV}$. In the absorber, the standard deviation depends on the measured energy. For instance its value is $11.4 \mathrm{keV}$ at $511 \mathrm{keV}$.

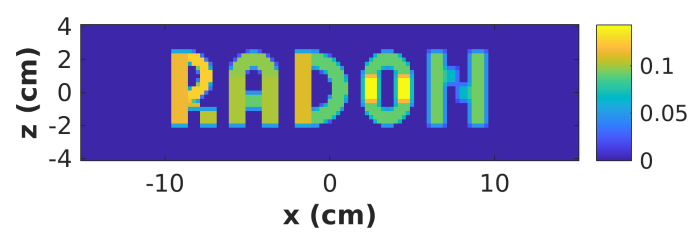

Figure 8: The reference image - one slice from a $1 \mathrm{~cm}$ thick source representing the word RADON.

The non-uniform source intensity has the form of the word RADON, with a thickness of $1 \mathrm{~cm}$ in the third direction (see figure 8). A total number of $10^{9} \gamma$ particles was emitted with energies $511 \mathrm{keV}$. After discarding not Compton and not reconstructible events (see conditions on the angles $\alpha$ and $\beta$ in section $\S 3.2$ ), about $17 \times 10^{6}$ reconstructible events were selected in the ideal case and about $8.1 \times 10^{6}$ in the realistic case.

The data were grouped in a five-dimensional array. The dimensions correspond to the layer, the azimuthal angle $\alpha$, the polar angle $\delta$, the parameter $\tau$ and the coordinate $s$ in the Radon projection of the Compton projection $\mathscr{P}_{\tau, \delta}$. The resulting array had $6 \times 45 \times 90 \times 45 \times 170$ elements. The higher the sampling density for the angular parameters, the better the reconstructed image should be as the sampling errors produce blur and artefacts that are much more severe than in imaging modalities based on line projections (see figure 7).

\section{Results and discussions}

Volumes having $120 \times 120 \times 33$ cubic voxels with side of $2.5 \mathrm{~mm}$ were reconstructed from ideal and noisy data. The resolution in the direction perpendicular to the camera is known to be worse than in slices parallel to it. We thus considered two situations where the word was placed either parallel to the camera or orthogonal to it. We also tested a reduction of the number of counts by a factor ten.

We compare three reconstruction methods: analytic stands for equation (32), Laplacian stands for (33) and semi-iterative stands for the algorithm described in paragraph 3.3.1. 


\subsection{Resolution in slices parallel to the detector}

In this test we place the word-shaped source parallel to the detector with the center at altitude $z=0$. The central slice is thus at $5 \mathrm{~cm}$ from the detector.

The first example is made with ideal data. We recall that ideal data include however some errors on energies induced by Doppler broadening and also statistical errors coming from the random generation of particles in the source. Even in this case, the noise in the reconstructed images is consequent as it may be seen in figure 9. Some denoising is necessary and we suggest that TV denoising would be a good choice in order to preserve the sharp edges of the source.

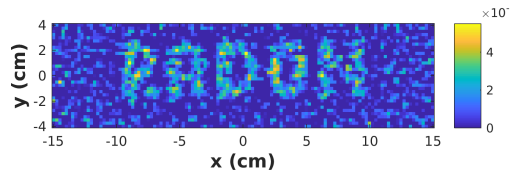

(a) analytic

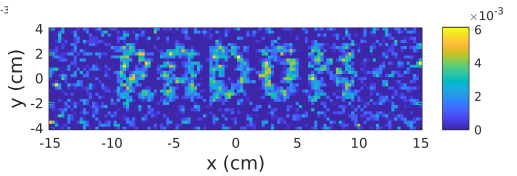

(b) Laplacian

Figure 9: Central slices from the word-shaped source placed parallel to the detector. The data are processed with SIVAN, meaning that only mild noise is considered (see text for details). The analytic and Laplacian methods give similar and noisy results.

Figure 10 shows on the left and center the central cuts of the same volumes as in figure 9 but after TV denoising. On the right is shown the TV-regularized SIRT reconstruction. The results for the three methods are very similar although the analytic reconstruction is slightly blurry (see letters $\mathrm{R}$ and $\mathrm{O}$ ).

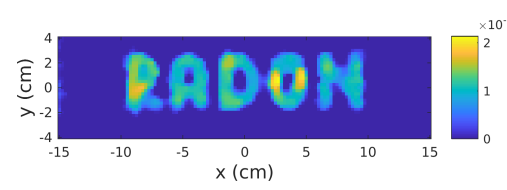

(a) analytic $(\mu=0.2)$

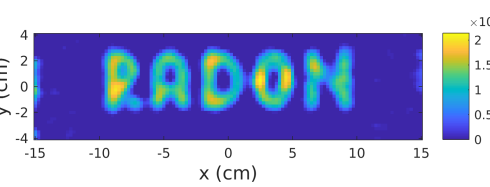

(b) Laplacian $(\mu=0.05)$

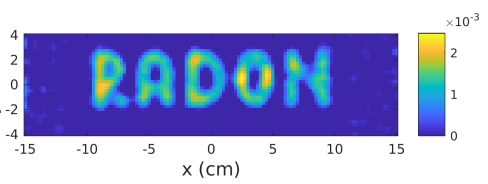

(c) semi-iterative $(\mu=0.01)$

Figure 10: The source is placed parallel to the detector and the collected events are ideal except the Doppler broadening. Central slices from the word-shaped source after TV denoising. For the semi-iterative method the result is shown after 30 iterations.

The evolution of the errors for the semi-iterative method is shown in figure 11 for the central slice. The figures of merit are the cost function from equation (5) displayed in blue and the root mean square error displayed in red. The iterations are started from the analytic solution.

When a realistic noise is added to the data, in practice by treating the output of the simulation with the module REVAN, the results are largely degraded but the shape of the letters is almost conserved (see figure 12). It should be noted however that the size of the voxel is here of $(2.5 \mathrm{~mm})^{3}$ which is very low when we know that the spatial 


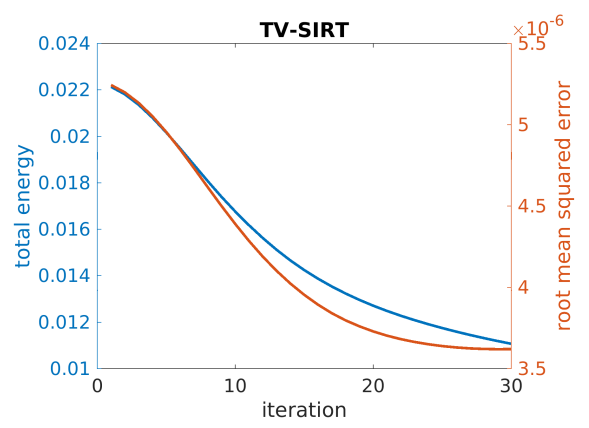

Figure 11: Evolution of the errors with the number of iterations for the regularized semi-iterative method. Ideal data, as produced in the output of the SIVAN module.

resolution currently obtained with collimated detectors in medical SPECT imaging is close to $5 \mathrm{~mm}$. Some degradation factors as attenuation in the patient and random coincidences were not considered in our experiment.

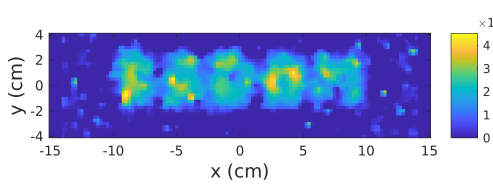

(a) analytic $(\mu=0.12)$

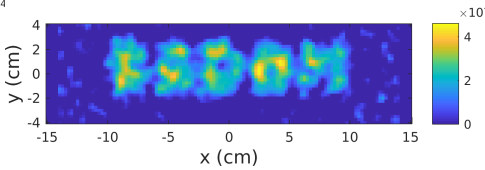

(b) Laplacian $(\mu=0.05)$

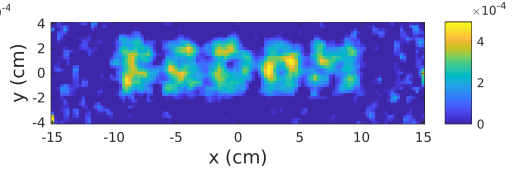

(c) semi-iterative $(\mu=0.01)$

Figure 12: Realistic data and TV denoising for the word-shaped source placed parallel to the detector. For the semi-iterative method the result is shown after 30 iterations.

Figure 13 shows the evolution of the errors for the semi-iterative method with realistic data.

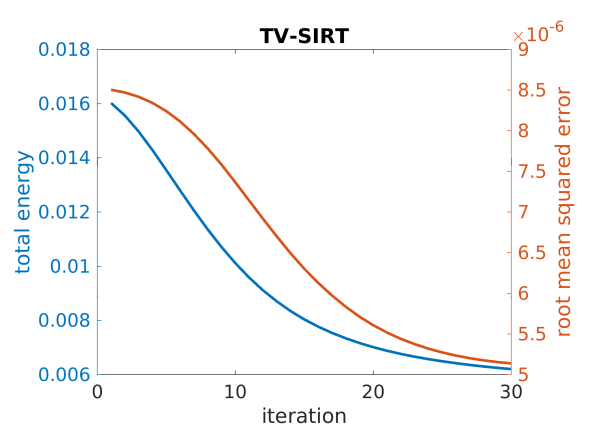

Figure 13: Evolution of the errors with the number of iterations for the regularized semiiterative method. Realistic data, as produced in the output of the REVAN module.

\subsection{Low statistics acquisition}

The quantity of available data has a strong influence on the noise as it is shown in figure 14. The image is reconstructed from a sub-set of $1.6 \times 10^{6}$ ideal events taken from the 
previous simulation (thus about one tenth of the sample).

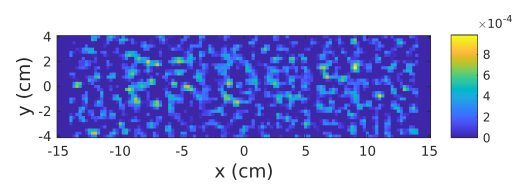

Figure 14: Low statistics reconstruction from ideal data, with the analytic formula.

Again, imposing low total variation to the reconstructed image helps to strongly improve the result. It may be noted in figure 15 that in this case the methods Laplacian and semi-iterative seem to outweigh the analytic method and to give sharper results.

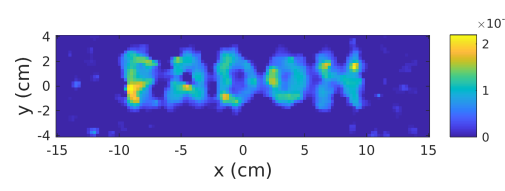

(a) analytic $(\mu=0.15)$

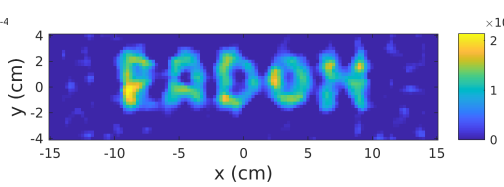

(b) Laplacian $(\mu=0.07)$

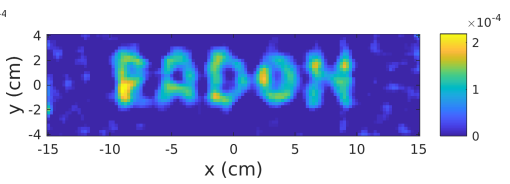

(c) semi-iterative $(\mu=0.02)$

Figure 15: Low statistics acquisition. Central slices from the word-shaped source placed parallel to the detector after TV denoising. The data are processed with SIVAN. For the semi-iterative method the result is shown after 20 iterations.

\subsection{Resolution in slices perpendicular to the detector}

In this test we place the word-shaped source perpendicular to the detector. The bottom of the source is at $3 \mathrm{~cm}$ from the up-most scatterer. The central slice refers in this case to $y=0$ and this slice is thus perpendicular to the detector, too. A blurring in the vertical direction can be observed. This blurring is usual in Compton camera imaging regardless the reconstruction method and uses to be attributed to projections loss due to the finite size of the detector. At our knowledge, the only theoretical study on the artifact produced by the data incompletness is the one from [29], where a "plume"shaped artifact is mentionned but under the convolutional conical Radon transform model. In section 4.1 we showed that the measurement errors and the binning are additional degradation factors. The relative influence the geometry and the errors on the angular parameters may have is an important question not yet elucidated.

The first test is done with ideal data and the results are displayed in figure 16. Let us mention that even ideal data are affected by the Doppler broadening that induce errors on the Compton angle. Those errors have a bell-shaped distribution with full width at half maximum of a few degrees, the exact value depending on the material and on the temperature. None of the methods presented here could retrieve the shape of the source when realistic data was used. The result is in all cases blurry and noisy and only the rough location of the word can be established. Again, the results from figure 17 show that the analytic method give the blurriest results. 


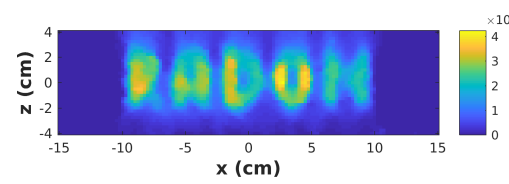

(a) analytic $(\mu=0.15)$

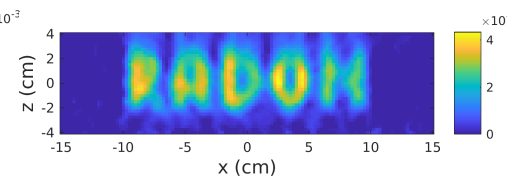

(b) Laplacian $(\mu=0.04)$

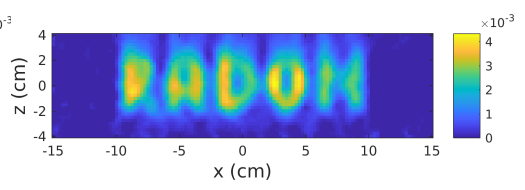

(c) semi-iterative $(\mu=0.01)$

Figure 16: Word perpendicular to the camera and ideal data from SIVAN output. Central slices after TV denoising of the volume. For the semi-iterative method the result is shown after 20 iterations.

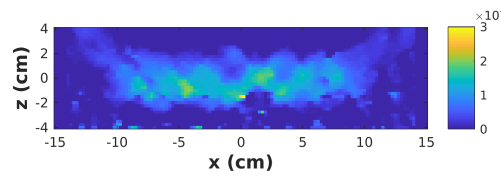

(a) analytic $(\mu=0.15)$

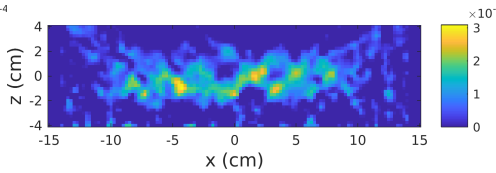

(b) Laplacian $(\mu=0.04)$

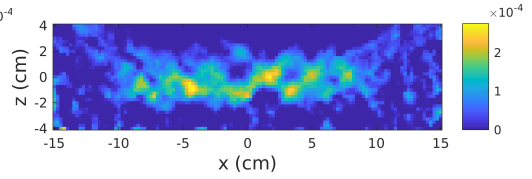

(c) semi-iterative $(\mu=0.01)$

Figure 17: Word perpendicular to the camera and realistic data from REVAN output. Central slices after TV denoising of the volume. For the semi-iterative method the result is shown after 30 iterations.

\section{Conclusions}

The purpose of this work is to push the limit of the attainable resolution in Compton camera imaging. We developed an inverse for a conical Radon transform and we tested three implementations for its numerical calculation. We demonstrated that imposing low total variation to the reconstructed volume strongly improves the result even when the level of the noise in the image is high. The results given by the three methods were very similar for ideal high statistics data. When the quality of the data drops, the Laplacian method, where the derivation is applied to the volume instead of the projections, and the semi-iterative method gave slightly better results in terms of sharpness. The best solution both in time spent in the reconstruction and quality of the image seems to be the Laplacian method. In the less favourable situation where the resolution in the vertical direction is tested with noisy data we did not succeeded to reconstruct an acceptable image of the source. The errors on the axes directions, on the half-opening angle and on the sequence of interactions, cumulated with the approximations induced by the binning, prevent reconstructing a source with small details as the one we chose for our tests. It should be noted however that we did not applied here clever data processing, consisting e.g., to select only events that have deposited in the detector a total energy close to the known initial energy of the photon. Our idea was to explore the limits of the methods, and in some situations the initial energy is unknown. 


\section{Acknowledgements}

The author acknowledges the financial support of the French National Research Agency through the ANR project 3DCLEAN (ANR-15-CE09-0009) and the LABEX PRIMES (ANR-11-LABX-0063) of Université de Lyon within the program "Investissements d'Avenir" (ANR-11-IDEX-0007) operated by the ANR. The author would like to thank the anonymous reviewers for careful reading of the manuscript and thoughtful comments.

\section{References}

[1] M. Schönfelder, A. Hirner, and K Schneider. A telescope for soft gamma ray astronomy. Nuclear Instruments and Methods, 107(2):385-394, 1973.

[2] R. Todd, J. Nightingale, and D. Everett. A proposed Gamma camera. Nature, 251:132-134, 1974.

[3] M. Singh and D. Doria. An electronically collimated gamma camera for single photon emission computed tomography. Part II: Image reconstruction and preliminary experimental measurements. Medical Physics, 10(4):428-435, 1983.

[4] E.A. Wulf, B.F. Phlips, W.N. Johnson, J.D. Kurfess, E.I. Novikova, P. O'Connor, and G. De Geronimo. Compton imager for detection of special nuclear material. Nuclear Instruments and Methods in Physics Research Section A: Accelerators, Spectrometers, Detectors and Associated Equipment, 579(1):371-374, 2007.

[5] W. Lee and T. Lee. A compact Compton camera using scintillators for the investigation of nuclear materials. Nuclear Instruments and Methods in Physics Research Section A: Accelerators, Spectrometers, Detectors and Associated Equipment, 624(1):118-124, 2010.

[6] S. Takeda, A. Harayama, Y. Ichinohe, H. Odaka, S. Watanabe, T. Takahashi, H. Tajima, K. Genba, D. Matsuura, H. Ikebuchi, Y. Kuroda, et al. A portable Si/CdTe Compton camera and its applications to the visualization of radioactive substances. Nuclear Instruments and Methods in Physics Research Section A: Accelerators, Spectrometers, Detectors and Associated Equipment, 787:207-211, 2015.

[7] C.G. Wahl, W.R. Kaye, W. Wang, F. Zhang, J.M. Jaworski, A. King, Y.A. Boucher, and Z. He. The Polaris-H imaging spectrometer. Nuclear Instruments and Methods in Physics Research Section A: Accelerators, Spectrometers, Detectors and Associated Equipment, 784:377-381, 2015.

[8] L.I. Rudin, S. Osher, and E. Fatemi. Nonlinear total variation based noise removal algorithms. Physica D: nonlinear phenomena, 60(1-4):259-268, 1992.

[9] P. Gilbert. Iterative methods for the three-dimensional reconstruction of an object from projections. Journal of theoretical biology, 36(1):105-117, 1972.

[10] V. Maxim. Filtered backprojection reconstruction and redundancy in Compton camera imaging. IEEE Transactions on Image Processing, 23(1):332-341, 2014.

[11] M. Fontana, D. Dauvergne, J.M. Letang, J.L. Ley, and É. Testa. Compton camera study for high efficiency SPECT and benchmark with Anger system. Physics in Medicine \& Biology, 62(23):8794, 2017.

[12] M. Frandes, A. Zoglauer, V. Maxim, and R. Prost. A tracking Compton-scattering imaging system for hadron therapy monitoring. IEEE Transactions on Nuclear Science, 57:144-150, 2010.

[13] S. Kurosawa, H. Kubo, K. Ueno, S. Kabuki, S. Iwaki, M. Takahashi, K. Taniue, N. Higashi, K. Miuchi, T. Tanimori, et al. Prompt gamma detection for range verification in proton therapy. Current Applied Physics, 12(2):364-368, 2012.

[14] E. Hilaire, D. Sarrut, F. Peyrin, and V. Maxim. Proton therapy monitoring by Compton imaging: influence of the large energy spectrum of the prompt- $\gamma$ radiation. Physics in Medicine \& Biology, 61(8):3127, 2016.

[15] S Aldawood, PG Thirolf, A Miani, M Böhmer, G Dedes, R Gernhäuser, C Lang, S Liprandi, 
L Maier, T Marinšek, et al. Development of a compton camera for prompt-gamma medical imaging. Radiation Physics and Chemistry, 140:190-197, 2017.

[16] E Muñoz, J Barrio, D Bemmerer, A Etxebeste, F Fiedler, F Hueso-González, C Lacasta, JF Oliver, K Römer, C Solaz, et al. Tests of macaco compton telescope with $4.44 \mathrm{mev}$ gamma rays. Journal of Instrumentation, 13(05):P05007, 2018.

[17] R. Basko, G. L. Zeng, and G. T. Gullberg. Application of spherical harmonics to image reconstruction for the Compton camera. Phys. Med. Biol., 43:887-894, 1998.

[18] L.C. Parra. Reconstruction of cone-beam projections from Compton scattered data. IEEE Transactions on Nuclear Science, 47(4):1543-1550, 2000.

[19] T. Tomitani and M. Hirasawa. Analytical image reconstruction of cone-beam projections from limited-angle Compton camera data. IEEE Trans. on Nuclear Science, 50(5):1602-08, October 2003.

[20] C-Y Jung and S. Moon. Exact inversion of the cone transform arising in an application of a Compton camera consisting of line detectors. SIAM Journal on Imaging Sciences, 9(2):520-536, 2016.

[21] M. J. Cree and P. J. Bones. Towards direct reconstruction from a gamma camera based on Compton scattering. IEEE Transactions on Medical Imaging, 13(2):398-407, 1994.

[22] M. Haltmeier. Exact reconstruction formulas for a Radon transform over cones. Inverse Problems, 30(3):035001, 2014.

[23] D. Schiefeneder and M. Haltmeier. The Radon transform over cones with vertices on the sphere and orthogonal axes. SIAM Journal on Applied Mathematics, 77(4):1335-1351, 2017.

[24] S. Moon. Inversion of the conical radon transform with vertices on a surface of revolution arising in an application of a Compton camera. Inverse Problems, 33(6):065002, 2017.

[25] S. Moon and M. Haltmeier. Analytic inversion of a conical Radon transform arising in application of Compton cameras on the cylinder. SIAM Journal on Imaging Sciences, 10(2):535-557, 2017.

[26] A. Kishimoto, J. Kataoka, T. Taya, L. Tagawa, S. Mochizuki, S. Ohsuka, Y. Nagao, K. Kurita, M. Yamaguchi, N. Kawachi, K. Matsunaga, H. Ikeda, E. Shimosegawa, and J. Hatazawa. First demonstration of multi-color 3-D in vivo imaging using ultra-compact Compton camera. Scientific Reports, 7, 2017.

[27] R. Gouia-Zarrad and G. Ambartsoumian. Exact inversion of the conical Radon transform with a fixed opening angle. Inverse Problems, 30(4):045007, 2014.

[28] F. Terzioglu. Some inversion formulas for the cone transform. Inverse Problems, 31(11):115010, 2015.

[29] Victor Palamodov. Reconstruction from cone integral transforms. Inverse Problems, 33(10):104001, 2017.

[30] B. Smith. Reconstruction methods and completeness conditions for two Compton data models. J. Opt. Soc. Am. A, 22:445-459, March 2005.

[31] P. Kuchment and F. Terzioglu. Three-dimensional image reconstruction from Compton camera data. SIAM Journal on Imaging Sciences, 9(4):1708 - 1725, 2016.

[32] P. Kuchment and F. Terzioglu. Inversion of weighted divergent beam and cone transforms. Inverse Problems \& Imaging, 11(6):1071-1090, 2017.

[33] Fatma Terzioglu, Peter Kuchment, and Leonid Kunyansky. Compton camera imaging and the cone transform: a brief overview. Inverse Problems, 34(5):054002, 2018.

[34] M.K. Nguyen and T.T. Truong. On an integral transform and its inverse in nuclear imaging. Inverse Problems, 18(1):265, 2002.

[35] V. Maxim, M. Frandes, and R. Prost. Analytical inversion of the Compton transform using the full set of available projections. Inverse Problems, 25(9):1-21, 2009.

[36] A. Sawatzky, C. Brune, F. Wubbeling, T. Kosters, K. Schafers, and M. Burger. Accurate EM-TV algorithm in PET with low SNR. In Nuclear Science Symposium Conference Record, 2008. NSS'08. IEEE, pages 5133-5137. IEEE, 2008.

[37] E.Y. Sidky, C.M. Kao, and X. Pan. Accurate image reconstruction from few-views and limited- 
angle data in divergent-beam CT. Journal of X-ray Science and Technology, 14(2):119-139, 2006.

[38] M Persson, D Bone, and $\mathrm{H}$ Elmqvist. Total variation norm for three-dimensional iterative reconstruction in limited view angle tomography. Physics in Medicine \& Biology, 46(3):853, 2001.

[39] A. Chambolle. An algorithm for total variation minimization and applications. Journal of Mathematical imaging and vision, 20(1-2):89-97, 2004.

[40] A. Beck and M. Teboulle. A fast iterative shrinkage-thresholding algorithm for linear inverse problems. SIAM journal on imaging sciences, 2(1):183-202, 2009.

[41] O. Klein and Y. Nishina. Über die streuung von strahlung durch freie elektronen nach der neuen relativistischen quantendynamik von dirac. Zeitschrift für Physik, 52(11-12):853-868, 1929.

[42] A. Zoglauer, R. Andritschke, and F. Schopper. MEGAlib-The medium energy gamma-ray astronomy library. New Astron. Rev., 50:629-632, October 2006.

[43] S. Agostinelli, J. Allison, K. Amako, J. Apostolakis, and al. Geant4-A simulation toolkit. Nucl. Instrum. Methods A, 506:205-303, 2003.

[44] S.J. Wilderman, W.L. Rogers, G.F. Knoll, and J.C. Engdahl. Fast algorithm for list mode backprojection of Compton scatter camera images. IEEE Transactions on Nuclear Science, 45:957962, June 1998.

[45] V. Maxim, X. Lojacono, E. Hilaire, J. Krimmer, E. Testa, D. Dauvergne, I. Magnin, and R. Prost. Probabilistic models and numerical calculation of system matrix and sensitivity in list-mode MLEM 3D reconstruction of Compton camera images. Phys. Med. Biol., 61(1):243-264, 2016.

[46] F. Natterer. The mathematics of computerized tomography, volume 32. Siam, 1986. 